\title{
Commentary
}

\section{Oxidative Damage and Cancer}

\author{
Terry D. Oberley \\ From the Department of Pathology and Laboratory Medicine, \\ University of Wisconsin Medical School and Veteran's \\ Administration Hospital, Madison, Wisconsin
}

Considerable evidence has linked oxidative damage and cancer. This Commentary will briefly review this area in light of new data linking specific gene modification with oxidative damage.

\section{Reactive Oxygen Species (ROS)}

ROS are generated in mitochondria of normal mammalian cells as a byproduct of normal respiration and in other subcellular locations as a function of biochemical reactions using oxygen. ${ }^{1}$ ROS at high levels are toxic to the cell, but at low levels, ROS have physiological functions, including activation and modulation of signal transduction pathways, ${ }^{2}$ modulation of activities of redox-sensitive transcription factors, ${ }^{3,4}$ and regulation of mitochondrial enzyme activities. ${ }^{5}$ Levels of ROS are reduced by antioxidant defenses, but increased by transition metals such as iron or copper and by exogenous agents such as ionizing radiation or ozone.

\section{Antioxidant Defenses}

To protect against toxic effects of ROS and to modulate physiological effects of ROS, the cell has developed an intricately regulated antioxidant defense system. The antioxidant enzyme system is very complex, being composed of small molecular weight antioxidant compounds (vitamins E, C, A, and so forth); primary (manganese, copper, zinc superoxide dismutases, catalase, glutathione peroxidase) and secondary antioxidant enzymes (enzymes such as glutathione reductase and glucose-6phosphate dehydrogenase); and the glutathione, ${ }^{6}$ glutaredoxin, ${ }^{7}$ and thioredoxin ${ }^{8}$ systems. Protein and DNA repair enzymes may be considered part of the antioxidant system. Proteins that sequester metals are important in modulating cell redox state. Nitric oxide modulates levels of ROS in part by its reaction with superoxide anion. Finally, proteins involved in response to cell stressors, such as the heat shock system, are important in modulating oxidant damage. Each component of the antioxidant system is specifically compartmentalized to specific subcellular locations. ${ }^{9,10}$

\section{Identification of Oxidative Damage}

Methods for identification of oxidative damage have recently been reviewed by Toyokuni. ${ }^{11}$ Just as the identification of antioxidant enzymes proved the existence of ROS, the presence of DNA repair enzymes specific for oxidative DNA damage documented the inherent toxicity of ROS for cellular DNA. ${ }^{12}$ In general, ROS have a short half-life because of their high reactivity and are therefore difficult to detect with standard biochemical methods. Advanced physical-chemical techniques such as electron spin resonance with spin traps allow detection and identification of free radicals. However, these techniques require expensive instrumentation, and quantification of the amount of oxygen-free radicals in tissues is difficult. ROS can be detected by methods allowing detection of fluorescence signals, eg, luciferase assays with a fluorometer or use of redox-sensitive dyes with flow cytometry or confocal microscopy. These fluorescent techniques suffer from a lack of specificity, but have high sensitivity. Histochemical techniques for identification of oxidant stress have been described, and these techniques have recently been reviewed; ${ }^{13}$ an example is the direct Schiff's reaction for the localization of lipid peroxidation in tissues.

Because of the caveats of the aforementioned techniques, investigators have turned to analyzing the footprints of oxidative damage, eg, analysis of oxidative damage products, including products of lipid, protein, and DNA oxidation. Oxidative damage products can be measured with biochemical assays. Antibodies to oxidative damage products have been developed and may be used in either biochemical or morphological assays. In addition, nitrosative damage can be detected with antibody to nitrotyrosine.

\footnotetext{
Accepted for publication November 27, 2001

Address reprint requests to Terry D. Oberley M.D., Ph.D., Department of Pathology and Laboratory Medicine, William S. Middleton Memorial VA Hospital, 2500 Overlook Terrace (Room A-35), Madison, WI 53705. Email: toberley@facstaff.wisc.edu.
} 


\section{Analysis of Antioxidant Enzymes and Oxidative Damage Products in Human Cancer}

It has been demonstrated both in vivo and in vitro that antioxidant enzyme levels are altered in cancer. The most consistent finding in biochemical studies has been that manganese superoxide dismutase (MnSOD), a mitochondrial antioxidant enzyme, is lowered in most types of primary cancers and cancer cell lines examined. ${ }^{14}$ Our laboratory has focused on antioxidant enzymes in human lung, ${ }^{15}$ renal, ${ }^{16,17}$ and prostate cancers; ${ }^{18,19}$ immunoperoxidase studies demonstrated low levels of antioxidant enzymes in primary tumors, although small groups of cancer cells, often on the invading edge of the tumor, did occasionally show strong positivity. These results agree with the well-developed paradigm from numerous biochemical studies that MnSOD levels are low in cancer. However, primary cancers from some organs have been shown to have high levels of MnSOD. In all cases, after comparison to normal tissue controls, MnSOD has been shown to be altered (activities elevated or depressed) in primary cancers. In our laboratory, metastatic prostate cancer was shown to have significantly elevated levels of MnSOD. ${ }^{19}$ One possible explanation of this finding is that primary prostate cancer cells undergo a selection process, with those cells destined for metastasis having high levels of MnSOD. Future studies will be necessary to test this hypothesis.

Some progress has been made in understanding the reason for abnormal regulation of MnSOD in cancer. Polymorphisms in the leader sequence of the MnSOD gene have been correlated with susceptibility to breast cancer, ${ }^{20}$ whereas additional mutations have been identified in the $5^{\prime}$-untranslated region, although the significance of these latter mutations is not known. ${ }^{21}$ The coding sequences for both manganese and copper, zinc superoxide dismutases were not mutated in colon cancer $^{21}$ and prostate ${ }^{18}$ cancer, respectively, although a polymorphism in the enzyme has been described. ${ }^{22}$ Therefore, abnormalities in mRNA regulation or posttranslational modifications need to be studied more intensely to explain the altered levels of MnSOD in these forms of cancer. Additional studies of other cancers will be necessary to determine whether mutations in the coding region can be found. It has yet to be determined whether abnormal antioxidant enzyme regulation is because of abnormalities in gene expression or some more fundamental property of cancer cells, ie, degree of cell differentiation.

One possible consequence of antioxidant enzyme imbalance is alterations in cellular redox state. Several laboratories have analyzed changes in cell redox state in cancer. Okamoto and colleagues ${ }^{23}$ demonstrated with biochemical techniques that renal cell carcinomas showed higher levels of DNA oxidation compared with corresponding normal tissue controls, as determined by measurements of 8-hydroxy-2'-deoxyguanosine (8$\mathrm{OHdG}$ ). Using immunoperoxidase techniques with specific antibodies to 4-hydroxy-2-nonenal (4HNE)-modified proteins, our laboratory demonstrated significant lipid peroxidation products in clear cell variant of renal carcinoma ${ }^{24}$ but little lipid peroxidation in other types of renal cancer (papillary carcinoma, Wilms' tumor, and transitional cell carcinoma of the renal pelvis). Kondo and colleagues $^{25}$ used antibodies against 8-OHdG, 4HNEmodified proteins, and 3-nitro-L-tyrosine (3-NT) to demonstrate oxidative stress in human colorectal carcinoma, but not in adenoma. High levels of DNA oxidation were confirmed with high-performance liquid chromatography measurements.

Our laboratory has studied human prostate cancer with immunohistology techniques using specific antibodies to oxidative and nitrosative damage products and have demonstrated low levels of oxidative stress (as measured with immunoperoxidase techniques using antibodies to 4HNE-modified proteins), but high levels of nitrosative (3-NT) stress in primary prostate cancer compared to normal prostate epithelium. ${ }^{19}$ Focal areas of primary prostate cancer showed very high levels of 8-OHdG. In contrast, metastatic prostate cancer had higher levels of oxidative (4HNE-modified proteins and 8-OHdG) and nitrosative (3-NT) damage products than either primary cancer or normal prostate epithelium. These results confirm abnormalities in oxidative and nitrosative metabolism in one human cancer. Interestingly, Kondo and colleagues ${ }^{25}$ also demonstrated high levels of 8-OHdG and 3-NT in primary colon cancer, suggesting that this phenotype is present in more than one type of cancer. It is highly attractive to speculate that high levels of 8-OHdG and 3-NT are because of low levels of MnSOD, a possibility that is being currently tested in our laboratory in cell culture systems. In contrast to our study of human prostate cancer, ${ }^{19}$ the study of Kondo and colleagues ${ }^{25}$ in human colon cancer showed increased levels of $4 \mathrm{HNE}$-modified proteins in malignant compared to adjacent normal epithelium. Our laboratory has demonstrated that $4 \mathrm{HNE}$-modified proteins are present in both nucleus and mitochondria in human renal cancer. ${ }^{24}$ The amount of $4 \mathrm{HNE}$-modified proteins is thus specific to cell type, because each cell type will have varying number and size of mitochondria; thus, differences in mitochondrial number and metabolism may explain the differences in levels of $4 \mathrm{HNE}$-modified proteins in prostate versus colon cancer. In support of mitochondria being a major source of $4 \mathrm{HNE}$-modified proteins is the finding that human renal oncocytoma, which is a tumor with large numbers of mitochondria per cell, demonstrated large amounts of $4 \mathrm{HNE}-$ modified proteins in cell cytoplasm. ${ }^{24}$

A major criticism of many studies to date is that levels of antioxidant enzymes and oxidative damage products have not been determined in preneoplastic lesions. However, Kondo and colleagues ${ }^{25}$ examined human colon adenomas, which demonstrated low levels of oxidative damage products compared to colon carcinomas, suggesting that oxidative stress may be a feature of neoplastic lesions only. Future studies of other preneoplastic lesions will be necessary to determine the generality of this finding. 


\section{In Vitro Model of Oxidative Damage and Cancer}

In vitro models have studied the effects of oxidative damage on cancer cells, and so provide information about the biochemistry of cancer cells. Unfortunately, because the cancer phenotype is already present, little can be learned about the carcinogenesis process. However, in vitro models do allow studies of differences between normal, malignant, and derived metastatic cancer cells, and therefore may provide important information for the development of possible therapeutic regimens.

One of the most studied model systems is transfection of MnSOD cDNA into cancer cells. MnSOD is a mitochondrial antioxidant enzyme with both antioxidant (removal of superoxide anion) and pro-oxidant (enzymatic generation of hydrogen peroxide) functions. The conditions under which antioxidant or pro-oxidant functions predominate are not certain, but in either case the mitochondrial redox state is modulated by MnSOD overexpression. It has been demonstrated in vitro that many cancer cells produce hydrogen peroxide, ${ }^{26}$ and one possible explanation for this is that cancer cells could have low levels of hydrogen peroxide-detoxifying enzymes.

It has been shown that overexpression of MnSOD inhibits cell growth in vitro in at least 13 malignant cell types. $^{27}$ In two cell types, hydrogen peroxide was strongly implicated in growth inhibition because double transfection with MnSOD plus mitochondrial catalase or glutathione peroxidase reversed growth inhibition. Further, strong evidence for the enzymatic activity of MnSOD causing growth inhibition and not functions of the 3 '- or $5^{\prime}$-untranslated regions of the MnSOD gene was provided by studying a polymorphism of the subunit MnSOD interface that has low MnSOD activity in comparison with wild-type protein; transfection of the polymorphic MnSOD cDNA, resulting in low MnSOD activity compared to activity resulting from transfection with normal MnSOD cDNA, showed little growth inhibition. ${ }^{22}$

The cellular mechanism by which MnSOD inhibits tumor growth is not certain, because MnSOD overexpression has pleiotropic effects on cells, including modulation of transcription factors, ${ }^{3,4}$ signal transduction pathways, ${ }^{2}$ mitochondrial function, ${ }^{28}$ and microtubule organization. ${ }^{29}$ Numerous studies have demonstrated that the mechanism of growth inhibition does not involve cell death, ie, necrosis or apoptosis. In addition, studies in our laboratory have demonstrated that MnSOD inhibits cell-cycle progression. ${ }^{29,30}$ It has been well documented that ROS at low levels stimulate cell proliferation, whereas higher levels of ROS cause cell injury. Thus, effects of MnSOD on cell cycle probably reflect net antioxidant and pro-oxidant functions on each individual cell type. Because many cancer cells have low levels of hydrogen-peroxide detoxifying enzymes, inhibition of cell growth may reflect the effects of hydrogen peroxide on cell growth. In fact, our laboratory has demonstrated that prostate carcinoma cells (DU145) overexpressing MnSOD have cell growth rates that correlate with $\mathrm{ROS}$ levels. ${ }^{29}$

\section{In Vivo Model of Oxidative Damage and Cancer: Multistage Mouse Skin Carcinogenesis}

A multistage carcinogenesis model has been used to study skin cancer in the mouse for many years. ${ }^{31}$ Stages of this model have been defined as initiation, promotion, and progression. 7-12-dimethylbenz(a)-anthralene (DMBA) has been used as a tumor initiator, whereas 12-O-tetradecanoylphorbol-13-acetate (TPA) has often been used as a tumor promoter. Although DMBA is known to result in DNA damage with resultant mutations, the role of TPA in tumor promotion is less well understood, although it has been demonstrated that TPA binds to the enzyme protein kinase $\mathrm{C}$ and hence regulates phosphorylation within cells. ${ }^{32}$ However, TPA may have other roles in tumor promotion. Keratinocytes treated with TPA in vitro show increased levels of ROS, ${ }^{33}$ and SOD mimetics, which reduce levels of superoxide anion, have been shown to reduce skin papilloma formation after DMBA/TPA treatment. $^{34}$

To study the role of a mitochondrial antioxidant enzyme in papilloma formation, transgenic mice overexpressing MnSOD have been developed. ${ }^{29}$ Transgenic mice that overexpressed MnSOD were shown to have reduced papilloma formation compared to their nontransgenic littermates. ${ }^{35}$ To study the relationship between DMBA/TPA treatment and oxidative damage, our laboratory analyzed oxidative damage products in keratinocytes from transgenic and nontransgenic mouse skin (data submitted). Nontransgenic and transgenic mice overexpressing MnSOD were topically treated with one dose (20 nmol/L) of DMBA and a subsequent dose $(4 \mu \mathrm{g})$ of TPA. At selected times after TPA treatment, mouse skin keratinocytes were analyzed for levels of MnSOD and levels of the oxidative damage product $4 \mathrm{HNE}$-modified proteins using specific antibodies and immunogold electron microscopy with computerized image analysis. At all time points analyzed after TPA treatment, there was more MnSOD immunoreactive protein in mitochondria of transgenic mouse keratinocytes treated with DMBA/TPA than in mitochondria of nontransgenic mouse keratinocytes similarly treated. To study the functional consequences of MnSOD overexpression, intact skin was analyzed for the presence of $4 \mathrm{HNE}-$ modified proteins. Compared to control groups, there was a large increase in $4 \mathrm{HNE}$-modified proteins at 6 to 24 hours after TPA in DMBA/TPA-treated mouse keratinocytes, and this increase was larger in nontransgenic than transgenic mice. Proteins modified by lipid peroxidation were detected in significant levels in keratinocyte mitochondria and nuclei, but at only low levels in cytoplasm. Mitochondrial injury was detected by electron microscopy as loss of cristae caused by inclusion formation; these inclusions were shown by specific antibody and immunogold techniques to contain 4HNEmodified proteins. The appearance of mitochondrial inclusions was delayed in keratinocytes of transgenic compared to nontransgenic mice. Thus, reduction in papilloma formation observed in transgenic mice overexpressing $\mathrm{MnSOD}$ is correlated with oxidative damage and mitochondrial events. 


\section{Oxidative Damage and Cancer: Iron Nitrilotriacetate (FeNTA)-Induced Rat Kidney Cancer}

FeNTA has been shown to cause kidney cancer in the rat. ${ }^{36}$ Presumably, iron is delivered to the proximal tubule by the nitrilotriacetate moiety of FeNTA. Once iron is present in nonprotein-bound forms in tissues, it can react in Fenton chemistry reactions to produce highly toxic ROS. Oxidative damage has been shown to be present in this model by the detection of lipid peroxidation products $^{37}$ and 8-hydroxy-2'-deoxyguanosine ${ }^{38}$ using both biochemical and immunohistology techniques with specific antibodies.

Studies in our laboratory have demonstrated accumulation of large amounts of 4 HNE-modified proteins in nuclei and mitochondria of proximal tubules of rat kidney after FeNTA treatment. ${ }^{39}$ Thus, although the oxidative damage seems to be cell-type-specific, oxidative damage appears in more than one subcellular compartment. It thus becomes difficult to understand specificity as a result of oxidative damage because injury is localized to more than one compartment of the cell. Because cancer is thought to involve sequential genetic changes in the nucleus, the question arises as to whether oxidative damage affects the genome equally or whether specific genes are targeted.

Analysis of renal cancers arising from FeNTA treatment has shown specificity in genes modified. No mutations were observed in $\mathrm{H}$-ras, $\mathrm{K}$-ras, and $\mathrm{N}$-ras oncogenes or p53 tumor suppressor gene in kidney cancer ${ }^{40}$ resulting from FeNTA treatment, whereas $p 15^{I N K 4 B}$ and $p 16^{I N K 4 A}$ tumor suppressor genes were shown to be inactivated. ${ }^{41}$ Because a prominent feature of cancer is chromosomal instability, it is difficult to determine whether genetic changes observed in cancer are causal in nature or simply a result of subsequent genetic changes. To begin to answer this question, two groups have analyzed genetic changes shortly after FeNTA treatment. Nomoto and colleagues $^{42}$ assessed the frequency of oxidative base damage hours after FeNTA treatment using the ligationmediated polymerase chain reaction technique. They assessed the frequency of 8-hydroxy-guanine (8-OH-Gua) formation in three genes, the tumor suppressor gene $p 53$, the heat shock protein 70 (HSP 70) gene, and the $\mathrm{Na}$, $K$-ATPase $\alpha 1$ subunit gene. Changes in $8-\mathrm{OH}$-Gua were not detected in p53 or HSP 70 genes after FeNTA treatment. In contrast, time-dependent alterations, corresponding to the time course of overall $8-\mathrm{OH}$-Gua formation and repair, were detected in the promoter region of the Na, K-ATPase $\alpha 1$ subunit gene. Thus, oxidative damage was not distributed uniformly along the whole genome, but seemed to be restricted to particular genes.

These results did not indicate which gene changes are actually important in the development of the rat renal cancer. In an article in the present issue of The American Journal of Pathology, Hiroyasu and colleagues ${ }^{43}$ determined whether oxidative damage can cause allelic loss 1 to 3 weeks after FeNTA administration. Using fluorescent in situ hybridization with imprint cytology at single cell resolution, these investigators found that the number of renal tubular cells with aneuploidy (one or three fluorescent signals as opposed to the expected two signals) at the $p 16^{\text {INK4A }}$ locus was specifically increased 1 to 3 weeks after administration of FeNTA. No increase in aneuploidy was observed at the loci of either the p53 or the $v$ hl tumor suppressor genes. Therefore, the $p 16^{I N K 4 A}$ loss is specifically vulnerable to oxidative damage, leading to its allelic loss within weeks.

\section{Specificity of Oxidative Damage Reactions}

Oxidative damage results from relatively nonspecific chemical reactions, yet, Hiroyasu and colleagues ${ }^{43}$ demonstrate specificity of gene damage. How can this occur? Most importantly, the specificity probably results from the specialized and localized redox environment of each cell type. That is, reactions generating pro-oxidants occur in specific locations within cells, antioxidant defenses are localized in specific subcellular compartments, and each cell type has unique redox state biochemistry. As a few examples, plasma membranes generate superoxide anion via NADPH oxidase, whereas smooth endoplasmic reticulum generates ROS via reactions involving cytochromes P450. Rough endoplasmic reticulum actually has a reducing environment. Antioxidant enzymes are localized in varying locations in cells. ${ }^{9} \mathrm{MnSOD}$ is in mitochondria, whereas CuZnSOD is located in cytoplasm, including lysosomes, and nucleus. Catalase is located primarily in peroxisomes, whereas glutathione peroxidase is found in all subcellular compartments. In addition, each mammalian cell type has unique amounts of antioxidant enzymes. As just one example, kidney proximal tubules have high levels of mitochondrial MnSOD, whereas kidney glomerular cells immediately adjacent have low levels of mitochondrial MnSOD. ${ }^{9}$ Components of the glutathione, ${ }^{6}$ glutaredoxin, ${ }^{7}$ and thioredoxin ${ }^{10}$ systems are also specifically compartmentalized within the cell. The unique amount and distribution of individual antioxidant proteins and compounds are a major determinant of the large variation in susceptibility of individual cell types to oxidative damage.

ROS generated also have different chemical properties. Thus, hydroxyl radical is so reactive that it immediately reacts with cellular components and thus cannot reach distant subcellular targets. Hydrogen peroxide and nitric oxide are much less reactive and more able to diffuse throughout the cell. Chemical reactions that ROS can participate in generate different products that form derivatives with varying degrees of toxicity. It must be re-emphasized that ROS and nitric oxide at low concentrations perform physiological functions, whereas at high concentrations these compounds are toxic.

Understanding the specificity of reactions will thus require a knowledge of the localized redox environment of each cell type and a more complete understanding under which specific oxidative and nitrosative chemical reactions occur. Such studies will form the basis for a better understanding of specific oxidative damage reactions. 


\section{Oxidative Damage and the Genome}

Understanding how specific genes are inactivated after oxidative damage is of great interest and importance. Possibilities for explaining specific gene loss include physical gene structure, chromosomal location, and/or physiological function of the gene. The latter possibility is especially intriguing because evidence is accumulating that genes modulating cell redox are, in turn, modulated by redox-regulated proteins that modulate specific mRNA stability. ${ }^{44}$ It is also known that redox state regulates specific transcription factors. ${ }^{3,4}$ It is possible that redox modulation may control accessibility of ROS to DNA. For example, ROS may cause removal of proteins from DNA and thus allow ROS attack of DNA.

\section{Conclusions}

Oxidative damage has long been implicated in both the malignant phenotype and carcinogenesis. Enthusiasm for these correlative studies has been tempered by the perceived lack of specificity of chemical reactions involving oxidative damage. In fact, specificity is probably achieved by the localized redox state biochemistry within each cell type. Understanding this localized redox microenvironment will be an exciting challenge for the future.

\section{References}

1. Chance B, Sies H, Boveris A: Hydroperoxide metabolism in mammalian organs. Physiol Rev 1979, 59:527-605

2. Monteiro HP, Stern A: Redox modulation of tyrosine phosphorylationdependent signal transduction pathways. Free Radic Biol Med 1996, 21:323-333

3. Li JJ, Oberley LW, Fan M, Colburn NH: Inhibition of AP-1 and NF- $\kappa B$ by manganese-containing superoxide dismutase in human breast cancer cells. FASEB J 1998, 12:1713-1723

4. Manna SK, Zhang HJ, Yan T, Oberley LW, Aggarwal BB: Overexpression of manganese superoxide dismutase suppresses tumor necrosis factor-induced apoptosis and activation of nuclear transcription factor- $\kappa$ B and activated protein-1. J Biol Chem 1998, 273:13245-13254

5. Nulton-Persson AC, Szweda LI: Modulation of mitochondrial function by hydrogen peroxide. J Biol Chem 2001, 276:23357-23361

6. Meister A, Anderson ME: Glutathione. Annu Rev Biochem 1983, 52: 711-760

7. Lundberg M, Johansson C, Chandra J, Enoksson M, Jacobson G, Ljung J, Johansson M, Holmgren A: Cloning and expression of a novel human glutaredoxin (Grx2) with mitochondrial and nuclear isoforms. J Biol Chem 2001, 276:26269-26275

8. Holmgren A: Thioredoxin. Annu Rev Biochem 1985, 54:237-271

9. Muse KE, Oberley TD, Sempf JM, Oberley LW: Immunolocalization of antioxidant enzymes in adult hamster kidney. Histochem J 1994, 26:734-753

10. Oberley TD, Verwiebe E, Zhong W, Kang SW, Rhee SG: Localization of the thioredoxin system in normal rat kidney. Free Radic Biol Med 2001, 30:412-424

11. Toyokuni S: Reactive oxygen species-induced molecular damage and its application in pathology. Pathol Int 1999, 49:91-102

12. Slupska MM, Baikalov C, Luther WM, Chiang JH, Wei YF, Miller JH: Cloning and sequencing a human homolog (hMYH) of the Escherichia coli mutY gene whose function is required for the repair of oxidative DNA damage. J Bacteriol 1996, 178:3885-3892

13. Frank J, Pompella A, Biesalski HK: Histochemical visualization of oxidant stress. Free Radic Biol Med 2000, 29:1096-1105

14. Oberley TD, Oberley LW: Antioxidant enzyme levels in cancer. Histol Histopathol 1997, 12:525-535
15. Coursin DB, Cihla HP, Sempf JM, Oberley TD, Oberley LW: An immunohistochemical analysis of antioxidant and glutathione S-transferase enzyme levels in normal and neoplastic human lung. Histol Histopathol 1996, 11:851-860

16. Oberley TD, Sempf JM, Oberley MJ, McCormick ML, Muse KE, Oberley LW: Immunogold analysis of antioxidant enzymes in human renal carcinoma. Virchows Arch 1994, 424:155-164

17. Oberley TD, Sempf JM, Oberley LW: Immunogold analysis of antioxidant enzymes in common renal tumors. Histol Histopathol 1996, 11:153-160

18. Bostwick D, Alexander E, Singh R, Shan A, Qian J, Santella R, Oberley LW, Yan T, Zhong W, Jiang X, Oberley TD: Antioxidant enzyme expression and reactive oxygen species damage in prostatic intraepithelial neoplasia and cancer. Cancer 2000, 89:123-134

19. Oberley TD, Zhong W, Szweda LI, Oberley LW: Localization of antioxidant enzymes and oxidative damage products in normal and malignant prostate epithelium. The Prostate 2000, 44:144-155

20. Ambrosone CB, Freudenheim JL, Thompson PA, Bowman E, Vena JE, Marshall JR, Graham S, Laughlin R, Nemoto T, Shields PG: Manganese superoxide dismutase (MnSOD) genetic polymorphisms, dietary antioxidants, and risk of breast cancer. Cancer Res 1999, 59:602-606

21. Xu Y, Krishnan A, Wan SX, Majima H, Yeh CC, Ludeweg G, Kasarskis EJ, St. Clair DK: Mutations in the promoter reveal a cause of the reduced expression of the human manganese superoxide dismutase gene in cancer cells. Oncogene 1999, 18:93-102

22. Zhang HJ, Yan T, Oberley TD, Oberley LW: Comparison of the effects of two polymorphic variants of manganese superoxide dismutase on human breast MCF-7 cancer cell phenotype. Cancer Res 1999, 59: $6276-6283$

23. Okamoto K, Toyokuni S, Uchida K, Ogawa O, Takenawa J, Katehi Y, Kinoshita K, Hattori-Nakakuki Y, Hiai H, Yoshida O: Formation of 8-hydroxy-2'-deoxyguanosine and 4-hydroxy-2-nonenal-modified proteins in human renal-cell carcinoma. Int J Cancer 1994, 58:825829

24. Oberley TD, Toyokuni S, Szweda LI: Localization of hydroxynonenal protein adducts in normal human kidney and selected human kidney cancers. Free Radic Biol Med 1999, 27:695-703

25. Kondo S, Toyokuni S, Iwasa Y, Tanaka T, Onodera H, Hiai H, Imamura $\mathrm{H}$ : Persistent oxidative stress in human colorectal carcinoma, but not in adenoma. Free Radic Biol Med 1999, 271:401-410

26. Szatrowski TP, Nathan CF: Production of large amounts of hydrogen peroxide by human tumor cells. Cancer Res 1991, 51:794-798

27. Oberley LW: Anticancer therapy by overexpression of superoxide dismutase. Antiox Redox Signaling 2001, 3:461-472

28. Li N, Oberley TD, Oberley LW, Zhong W: Overexpression of manganese superoxide dismutase in DU145 human prostate carcinoma cells has multiple effects on cell phenotype. The Prostate 1998, 35:221-233

29. Li N, Zhai Y, Oberley TD: Two distinct mechanisms for inhibition of cell growth in human prostate carcinoma cells with antioxidant enzyme imbalance. Free Radic Biol Med 1999, 26:1154-1168

30. Li N, Oberley TD: Modulation of antioxidant enzymes, reactive oxygen species, and glutathione levels in manganese superoxide dismutaseoverexpressing $\mathrm{NIH} / 3 \mathrm{~T} 3$ fibroblasts during the cell cycle. J Cell Physiol 1998, 177:148-160

31. Boutwell RK: The function and mechanism of promoters of carcinogenesis. CRC Critical Reviews in Toxicology. Edited by L Goldberg. Cleveland, CRC Press, Inc., 1974, pp 419-443

32. Kikawa U, Takai Y, Tanaka Y, Miyake R, Nishizuka Y: Protein kinase $C$ as a possible receptor protein of tumor-promoting phorbol esters. J Biol Chem 1983, 258:11442-11445

33. Robertson FM, Beavis AJ, Oberyszyn TM, O'Connell TM, Dokidos A, Laskin DL, Laskin JD, Reiners JJ: Production of hydrogen peroxide by murine spidermal keratinocytes following treatment with the tumor promoter, 12-O-tetradecanoylphorbol-13-acetate. Cancer Res 1990, 58:6062-6067

34. Egner PA, Kensler TW: Effects of a biomimetic superoxide dismutase on complete and multistage carcinogenesis in mouse skin. Carcinogenesis 1985, 6:1167-1172

35. Zhao Y, Xue Y, Oberley TD, Kiningham KK, Lin S, Yen HC, Majima H, Hines J, St. Clair DK: Overexpression of MnSOD suppresses tumor 
formation by modulation of AP-1 signaling in a multistage skin carcinogenesis model. Cancer Res 2001, 61:6082-6088

36. Ebina Y, Okada S, Hamazaki S, Ogino F, Li JL, Midorikawa O: Nephrotoxicity and renal cell carcinoma after use of iron- and aluminum-nitrilotriacetate complexes in rats. J Natl Cancer Inst 1986, 76: 107-113

37. Toyokuni S, Uchida K, Okamoto K, Hattori-Nakakuki Y, Hiai H, Stadtman ER: Formation of 4-hydroxy-2-nonenal-modified proteins in the renal proximal tubules of rats treated with a renal carcinogen, ferric nitrilotriacetate. Proc Natl Acad Sci USA 1994, 91:2616-2620

38. Toyokuni S, Mori T, Dizdaroglu M: DNA base modifications in renal chromatin of Wistar rats treated with a renal carcinogen, ferric nitrilotriacetate. Int J Cancer 1994, 57:123-128

39. Zainal TA, Weindruch R, Szweda LI, Oberley TD: Localization of 4-hydroxy-2-nonenl-modified proteins in kidney following iron overload. Free Radic Biol Med 1999, 26:1181-1193

40. Nishiyama Y, Suwa H, Okamoto K, Fukumoto M, Hiai H, Toyokuni S: Low incidence of point mutations in $\mathrm{H}-, \mathrm{K}$-, and $\mathrm{N}$-ras oncogenes and p53 tumor suppressor gene in renal cell carcinoma and peritoneal mesothelioma of Wistar rats induced by ferric nitrilotriacetate. Jpn J Cancer Res 1995, 86:1150-1158

41. Tanaka $T$, Iwasa $Y$, Kondo S, Hiai H, Toyokuni S: High incidence of allelic loss on chromosome 5 and inactivation of $\mathrm{p} 15^{\mathrm{INK} 4 \mathrm{~B}}$ and p15 INK4A tumor suppressor genes in oxystress-induced renal cell carcinoma of rats. Oncogene 1999, 18:3793-3797

42. Nomoto M, Yamaguchi R, Kawamura M, Kohno K, Kasai H: Analysis of 8-hydroxyguanine in rat kidney genomic DNA after administration of a renal carcinogen, ferric nitrilotriacetate. Carcinogenesis 1999, 20:837-841

43. Hiroyasu M, Ozeki M, Kohda H, Echizenya M, Tanaka T, Hiai H, Toyokuni S: Specific allelic loss of p16 $6^{\text {INK4A }}$ tumor suppressor gene after weeks of iron-mediated oxidative damage during rat renal carcinogenesis. Am J Pathol 2002, 160:419-424

44. Knirsch L, Clerch LD: Tyrosine phosphorylation regulates manganese superoxide dismutase (MnSOD) RNA-binding protein activity and MnSOD protein expression. Biochemistry 2001, 40:78907895 